\title{
La resolución de problemas a través del diseño co-creativo.
}

\author{
aSantamarina-Campos, Virginia, 'de-Miguel-Molina, María, 'de-Miguel-Molina, \\ Blanca, ${ }^{\mathrm{b} C a r a b a l-M o n t a g u d, ~ M a r i ́ a-A ́ n g e l e s ~}$
}

${ }^{a}$ Departamento de Conservación y Restauración de Bienes Culturales, Universitat Politècnica de València, virsanca@crbc.upv.es ${ }^{\mathrm{b}}$ Departamento de Organización de Empresas, Universitat Politècnica de València, mademi@omp.upv.es, ${ }^{c}$ Departamento de Organización de Empresas, Universitat Politècnica de València, bdemigu@gmail.com y d Departamento de Conservación y Restauración de Bienes Culturales, Universitat Politècnica de València, macamon@,crbc.upv.es

\begin{abstract}
Cooperative learning, based on problem solving, through the design of mobile conceptual maps, provides clarity, helping to make the ideas visible, tangible and sequential. Mobile cards allow more efficient system models to be obtained by allowing repetition with greater fluency. On the other hand, group work with the use of mobile notes results in a richer systemic model, because it synthesizes different points of view, fusing mental models, and facilitating the task of understanding and solving complex problems in a cooperative, creative and innovative way.
\end{abstract}

Keywords: cooperative learning, teaching innovation, team work, conceptual maps, mental maps, mental models, mobile cards, problem analysis, problem solving, project design, EICE-AFA-Realidad.

\begin{abstract}
Resumen
El aprendizaje cooperativo, basado en la resolución de problemas, mediante el diseño de mapas conceptuales móviles, proporciona claridad, ayudando a hacer las ideas visibles, tangibles y secuenciales. Las tarjetas móviles, permiten obtener modelos de sistemas más eficaces, al permitir la reiteración con mayor fluidez. Por otro lado el trabajo en grupo con el empleo de notas móviles da como resultado un modelo sistémico más rico, porque sintetiza diferentes puntos de vista, fusionando modelos mentales, y facilitando la tarea de entender y resolver problemas complejos de forma cooperativa, creativa e innovadora.
\end{abstract}

Palabras clave: aprendizaje cooperativo, innovación docente, trabajo en grupo, mapas conceptuales, mapas mentales, modelos mentales, tarjetas móviles, análisis de problemas, resolución de problemas, diseño de proyectos, EICE-AFA-Realidad. 


\section{Introducción}

\subsection{Contextualización de la experiència}

El uso de notas móviles nos permite obtener modelos más eficientes y ricos para resolver problemas, ya que proporcionan una herramienta flexible para su análisis. Además, el diseño de mapas conceptuales móviles aporta claridad mostrando las ideas de una manera visual. Las secuencias del proceso pueden variarse, añadiendo ideas de una manera creativa, utilizando palabras clave o dibujos. Los objetivos y retos de cada sesión necesitan ser diseñados por anticipado para guiar a los alumnos durante el proceso de aprendizaje, independientemente de a qué área pertenezca la asignatura.

Por otro lado, se trabajan cuatro competencias transversales (ICE 2015):

- Trabajo en equipo y liderazgo: "implica crear y desarrollar un clima de confianza mutua entre los componentes que permita trabajar de forma responsable y cooperativa...compartir conocimientos, compromiso y responsabilidad. Supone el reparto de tareas y roles y el respeto a las normas y reglas de juego establecidas por y para el grupo".

- Planificación y gestión del tiempo: "implica ser capaz de organizar y distribuir correctamente el tiempo del que disponemos y distribuirlo en función de las actividades necesarias para alcanzar nuestros objetivos a corto, medio y largo plazo".

- Innovación, creatividad y emprendimiento: "La innovación se entiende como la capacidad de dar respuesta satisfactoria a las necesidades personales, organizativas y sociales, modificando procesos y/o resultados para generar nuevo valor. A su vez, el desarrollo de esta competencia requiere, tanto el pensar de otro modo para aportar distintas perspectivas (creatividad), como el comprometer determinados recursos por iniciativa propia, con el fin de explorar una oportunidad, asumiendo el riesgo que esto comporta (emprendimiento)".

- Análisis y resolución de problemas: "Los problemas son situaciones nuevas que requieren que los individuos respondan con comportamientos nuevos. Resolver un problema implica realizar tareas que demandan procesos de razonamiento más o menos complejo y, en muchas ocasiones, no simplemente una acción asociativa y rutinaria".

El uso de mapas conceptuales, teniendo claros los objetivos y la manera de evaluarse, son una herramienta muy potente para afianzar los conceptos clave y conseguir un alineamiento constructivo (Biggs 2004).

La metodologia se ha implementado en las siguientes asignaturas:

1. Nuevas Tendencias en Estrategia de Servicio (31985). Optativa. Número de ECTS: 5. $\mathrm{N}^{\mathrm{a}}$ de estudiantes: 30. Facultad Administración y Dirección de Empres, Master Universitario en Gestión de Empresas, Productos y Servicios.

2. Metodologías de investigación (32257). Optativa. Número de ECTS: 3. $\mathrm{N}^{\mathrm{a}}$ de estudiantes: 30. Facultad Administración y Dirección de Empresa. Master Universitario en Gestión de Empresas, Productos y Servicios.

3. Gestión del patrimonio inmaterial (33848). Optativa. Número de ECTS: 4,5. N de estudiantes: 35. Departamento de Conservación y Restauración de Bienes Culturales, Máster Universitario en Conservación y Restauración de Bienes Culturales. 
4. Desarrollo, gestión y dirección de proyectos de restauración. Optativa. Número de ECTS: 4,5. $\mathrm{N}^{\mathrm{a}}$ de estudiantes: 35 . Departamento de Conservación y Restauración de Bienes Culturales, Máster Universitario en Conservación y Restauración de Bienes Culturales.

5. Historia de la Conservación y restauración de bienes culturales (33841). Optativa. Número de ECTS: 4. $\mathrm{N}^{\mathrm{a}}$ de estudiantes: 35. Departamento de Conservación y Restauración de Bienes Culturales, Máster Universitario en Conservación y Restauración de Bienes Culturales.

\subsection{Justificación y motivación}

En el diseño de proyectos, la fase más importante es la de identificación, en la que se analizará la situación existente para crear una visión de la situación deseada y seleccionar las estrategias que se emplearán para lograrla. Hasta ahora una de las principales herramientas empleadas en el análisis de problemas para poder plantear una idea innovadora, ha sido el método dafo. Este método permite identificar los puntos fuertes y débiles de un área concreta y las amenazas y oportunidades del entorno, obteniendo una imagen global de la problemática del recurso patrimonial. A pesar de que esta técnica puede ayudar en la elaboración de estrategias o alternativas que mitiguen las debilidades a partir de las potencialidades y fortalezas existentes, no permite obtener una visión objetiva del problema focal, así como de sus causas y efectos. Actualmente la visualización de las relaciones causales de los problemas de una forma clara, supone una tarea fundamental. Por tanto la elaboración de un diagnostico que permita obtener un conocimiento profundo de la situación como base para la toma de decisiones, exige el empleo de una herramienta que permita entender porque se ha producido esa situación no desea y cuales con sus consecuencias, facilitando la priorización de los problemas y el llegar a un acuerdo colaborativo en el diagnóstico entre implicados (Santamarina Campos et al. 2016).

En este caso los mapas conceptuales móviles cooperativos, han permitido visualizar las relaciones causales, priorizar los problemas y llegar a un acuerdo en el diagnóstico entre implicados, a través del trabajo colaborativo. La posibilidad de trabajar con mapas móviles, ha permitido la revisión colaborativa hasta verificar que el sistema es valido, haciendo los ajustes necesarios y llegando a consenso sobre su estructura.

\section{Objetivos}

El objetivo de este es estudio, es analizar los cambios producidos en el rendimiento académico del alumno y su percepción de la materia, a través de la introducción del uso de los mapas conceptuales móviles y cooperativos para la resolución de problemas.

Para ello la dinámica planteada se focalizara en analizar un problema complejo y buscar soluciones utilizando los mapas conceptuales móviles, de manera que a través de preguntas de enfoque el alumno vaya de lo general a lo específico, para buscar una solución en equipo.

Asimismo, esta experiencia se enmarca dentro del Equipo de Innovación y Mejora Educativa (EICE) "El proceso de Enseñanza y Aprendizaje Fuera de las Aulas - Baños de Realidad (EICE- AFA-Realidad)", en el que participan profesores de otros centros de la UPV con los que trabajamos estas dinámicas. Y del Proyecto de Innovación y Mejora Educativa "La resoluciones de problemas a través del diseño creativo y la visualización colaborativa" (PIME A09). 


\section{Descripción del desarrollo de la innovación}

De acuerdo con el "Modelo para la enseñanza del diseño creativo" (Glen et al. 2015), podemos desarrollar distintas actividades:

1. Identificar problemas: a los equipos se les plantea un reto (o pregunta de enfoque) y deben identificar problemas y oportunidades.

2. Observación: pensando de la misma manera que el usuario.

3. Visualización y reflexión: mostrando los resultados de la observación a toda la clase de manera visual para compartir, hacer preguntas y explicar.

4. Brainstorming de ideas, votando las mejores para reducir las soluciones.

5. Usar y probar prototipos, observando a los usuarios para captar nuevas ideas.

6. Test de viabilidad, explorando las opciones de modelos de negocio y las distintas posibilidades de generar valor.

En este caso, se han desarrollado actividades de los tipos 1, 3 y 4. De todos modos, dependiendo de la asignatura o session se podrían añadir otras actividades. Por ejemplo, se podría hacer un juego de rol (role-playing) para aplicar las actividades 2 y/o 5 . O hacer un estudio de caso utilizando la actividad 6.

Antes de comenzar la dinámica, son importantes dos tareas por parte del profesor:

- Redactar unas instrucciones previas para los estudiantes.

- Comprar el material necesario. Nosotros compramos tarjetas de la marca post-it que nos permiten manejar distintos tamaños, colores y formas.

Los grupos se forman con 3-5 alumnos. Se les plantea un reto y ellos deben identificar los distintos problemas, expresándolos con palabras clave o dibujos en notas móviles de un color determinado.

De acuerdo con el proceso de resolución de problemas, los grupos buscarán soluciones para cada problema por medio de la tormenta de ideas (brainstorming), de forma que al final se seleccionará una solución consensuada para cada problema. Dicha solución se expresará también con palabras clave o dibujos en una nota móvil de otro color, y que se conectará con el problema a resolver. Es decir, de cada problema una nota y de cada solución otra nota. En este momento, el professor revisará los modelos de cada grupo para detector información que falte por completer.

Al mostrar este modelo con las notas móviles pegadas en la pared, tendremos dos filas de ideas, problemas y soluciones. Entonces se plantea un nuevo reto, que es proponer medidas para prevenir que esos problemas aparezcan. Estas medidas, se escribirán o dibujarán en una nota móvil mayor y con un tercer color.

Asimismo, se podría plantear un tercer reto que sería establecer otras conexiones entre problemas y soluciones. Es decir, conectar soluciones que pueden ser communes para solucionar varios problemas.

Con el modelo final, el resto de grupos se desplazan por el aula para observar los modelos del resto de equipos y así pueden comparar dichos modelos con el suyo propio. Pero la mitad del grupo se queda en su modelo para explicar dudas a los "visitantes" o recoger las "recomendaciones" de sus compañeros. Acabada la ronda, cada grupo vuelve a reunirse para enriquecer su modelo con todas las ideas aportadas (De Miguel Molina et al. 2017). 


\section{Resultados}

A partir de la digitalización de las distintas fases de los trabajos de los alumnos, se ha realizado un análisis cuantitativo de los nodos, enlaces, uso de imágenes o uso de palabreabas claves, y los resultados obtenidos han sido:

$1^{\circ}$. El $90 \%$ de los diseños siempre presentan nodos y enlaces.

$2^{\circ}$. Los diseños que combinan enlaces y nodos, muestran un nivel de complejidad en función del número de nodos que suelen ir entre 6 y 8 . Los que utilizan menos nodos son más sencillos de entender, mientras que los que emplean más de 13 dan como resultado mapas complejos que dificultan su comprensión. Por lo que el número adecuado de nodos en un mapa, si se quiere mostrar algo de forma visual, debería estar entre 5 y 13 .

En ambas facultades, podemos comprobar que las ideas pueden representarse por texto (palabras clave) o con dibujos, dependiendo del reto propuesto y los objetivos.

Este tipo de ejercicios visuales, les ayuda a organizar ideas y a entender las relaciones o "conectores" entre los problemas y las soluciones. Además, las notas móviles permiten hacer cambios y resolver errores de una manera más sencilla, a la vez que los colores ayudan a diferenciar los conceptos.

La integración de distintos puntos de vista de modo colaborativo es muy importante en la resolución de problemas, por lo que el resultado final es más rico, pero, al mismo tiempo, es sencillo de comprender. Además, les ayuda a reflexionar sobre la necesidad de tener en cuenta el punto de vista de distintos actores (Santamarina et al. 2018).

Por dicho motivo, durante el curso 2017-2018 se han elaborado tres manuales orientados a la comunidad universitaria, para poder llevar a cabo la resolución de problemas, y facilitar el obtener ideas innovadoras para productos o servicios, procesos o gestión. Los tres manuales se depositaron en septiembre de 2018 con licencia Creative Commons Attribution 4.0 International, en el repositorio europeo Zenodo, creado por OpenAIRE y CERN, y con el apoyo de la Comisión Europea. Los títulos de las guía de usuario sobre el uso de esta metodología son:

1. GENERACIÓN DE IDEAS ( $\left(1^{\mathrm{a}}\right.$ parte): Tengo una idea, ¿cómo la represento?. DOI 10.5281/zenodo. 1703795

http://doi.org/10.5281/zenodo. 1703795

2. GENERACIÓN DE IDEAS ( $2^{\mathrm{a}}$ parte): De la idea al proyecto.

DOI 10.5281/zenodo. 1704063

http://doi.org/10.5281/zenodo. 1704063

3. GENERACIÓN DE IDEAS ( $3^{\text {a }}$ parte): ¿Cómo convencer con valor?.

DOI 10.5281/zenodo. 1704140 .

http://doi.org/10.5281/zenodo. 1704140

La implementación de los mapas conceptuales colaborativos y el uso del lenguaje visual, ha producido cambios importante, tanto en el rendimiento académico de los alumnos, como en la adquisición de competencias. Esto queda reflejado no solo en las calificaciones finales de los alumnos, sino también en la actitud frete a la materia, que queda reflejada en las encuesta al profesorado.

Para analizar el impacto del uso de esta herramienta, se tomo como punto de control una de las materias en las que se implemento, en la cual se ha obtenido como principal resultado, una evolución en las calificaciones globales de las asignaturas (Imagen 8 y 9), habiéndose 
registrado un aumento en las calificaciones numéricas, que evidencian no solo el incremento de la implicación del alumnado con los contenidos de la asignatura, sino también una motivación que favorece el generar ideas innovadoras. Por otro lado, el promedio de la evolución de las calificaciones teóricas, se ha visto significativamente incrementada, dado que en el curso 2016/17 se obtuvo una media de 7,32 sobre 10 , mientras que en el curso académico 2017/18 el resultado fue del 9,09 sobre 10 en los contenidos trabjados a partir de mapas conceptuales. Este incremento se atribuye no solo al empleo de los mapas colaborativos, si no al empleo del lenguaje visual.

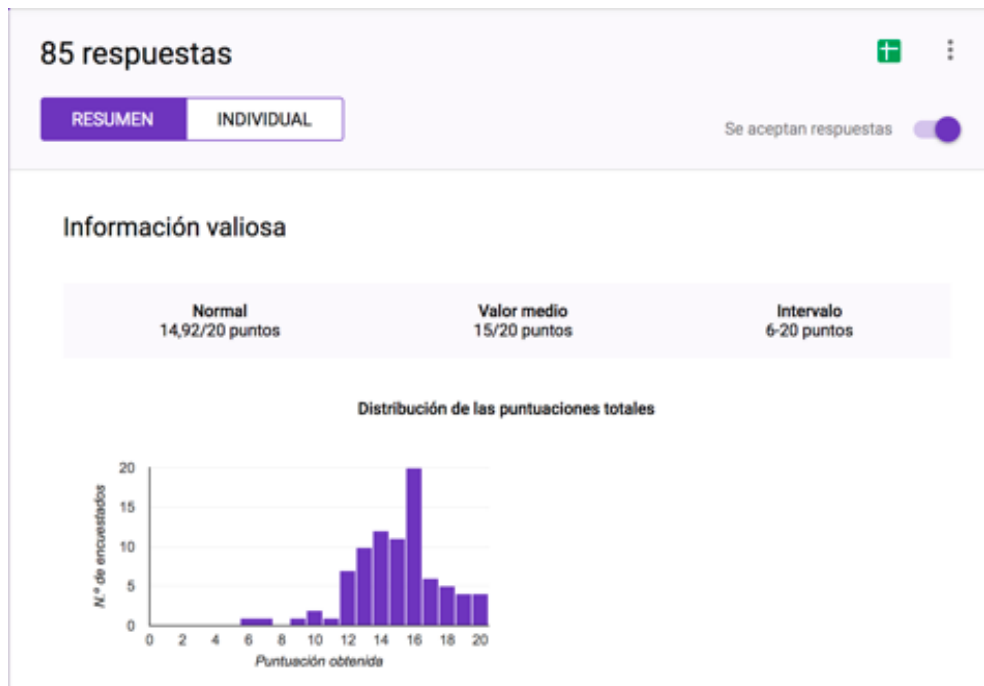

Imágenes 8. Resultados obtenidos curso 2016-17

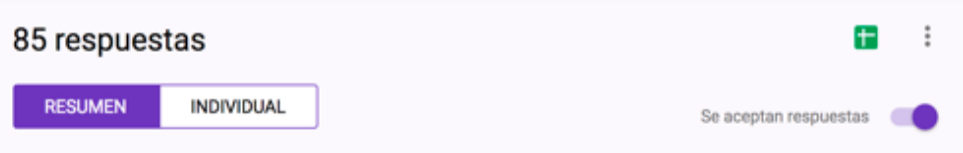

Información valiosa

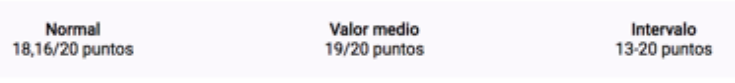

Distribución de las puntuaciones totales

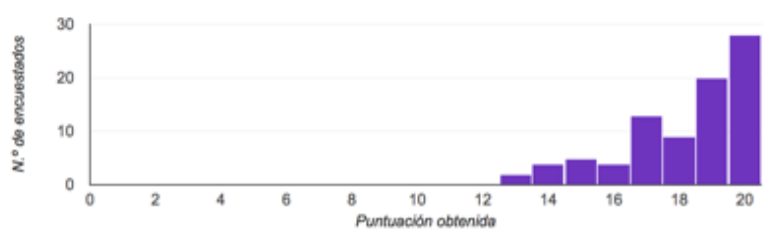

Imágenes 9. Resultados obtenidos curso 2017-18 


\section{Conclusiones}

El objetivo es continuar empleando esta metodología, a pesar de que supone un sobre esfuerzo importante para el docente. Sería importante que el trabajo de los profesores que implementan estas metodologias, se pudiera valorar en las encuestas al profesorado. La disposición y equipamiento de las aulas, dificulta la implementación de esta herramienta, dado que están preparadas para lecciones magistrales. Esto supone el mover mobiliario continuamente, buscar paredes donde poder colocar los trabajo, juntar mesas para poder trabajar sobre formatos grandes, etc.. Se debería de habilitar aulas experimentales en todos los centro que se adecuaran a las necesidades de metodologías más dinámicas y colaborativas, que propiciarán un ambiente de trabajo más creativo.

Esta experiencia nos ha mostrado que el uso de notas móviles ayuda a impulsar el trabajo colaborativo en distintas asignaturas, se usen palabras clave o dibujos. Y, evidentemente, el trabajo colaborativo es fundamental a la hora de resolver problemas (Arquilla and Motta 2011), ya que distintos actores pueden estar involucrados y las relaciones interpersonales son fundamentales (Muñoz González, Serrano Rodríguez, and Marín Díaz 2014). El intercambio de ideas enriquece la visión de los problemas y la generación de posibles soluciones.

Es cierto que los cursos de postgrado suelen tener grupos pequeños, por lo que estas dinámicas son más sencillas de aplicar y además ayudan a impulsar el trabajo colaborativo, a la vez que ayudan a gestionar el tiempo puesto que cada fase tiene un tiempo máximo para que la dinámica pueda completarse en la sesión. Y estas son competencias transversales o "soft" que demandan los empleadores (Wu-Pong et al. 2013), más allá de conocimientos específicos. Incluso si los estudiantes no son expertos en la materia, y vienen de titulaciones distintas, este trabajo cooperativo les ayuda a trabajar en equipos interdisciplinares.

Se abren distintas preguntas a la hora de evaluar este trabajo, puesto que es difícil marcar elementos objetivos que ayuden al profesor, siendo más una evaluación por observación en el aula, combinada con el resultado final. Algunas sugerencias podrían ser: valorar la integración del equipo, valorar la cantidad y calidad de ideas expuestas (problemas detectados y/o soluciones propuestas), valorar el número de conexiones entre los problemas y soluciones, valorar la capacidad de síntesis (a través de palabras clave o dibujos), valorar la creatividad a la hora de exponer el mapa, valorar el ajuste al tiempo dado, etc.

Como conclusiones finales podemos destacar tres puntos:

1. Los diferentes diseños, representan mapas conceptuales, que revelan puntos de vista diferentes. A pesar de no ser expertos en diseño de mapas conceptuales, logran de forma intuitiva dividir procesos complejos en tareas simples, organizándolo y sintetizarlo en un mapa.

2. La facilidad con la que se puede modificar un mapa, está vinculada con nuestro interés de mejorar el modelo. Así que un sistema con notas post-it no solo es más dinámico y flexible, sino que también genera más nodos que los dibujos realizados directamente sobre el papel.

3. Los mapas conceptuales colaborativos, logran integrar la diversidad de puntos de vista individuales de todos los alumnos. Estos nuevos sistemas contienen un elevado número de nodos, incluso nuevas ramificaciones y patrones, empleando diferentes colores de tarjetas, pero para los alumnos no resultan complejos porque participan en la construcción de estos modelos comunes. 


\section{Referencias}

Arquilla, V. and R. Motta. 2011. "Student Brain: Tools and Methods to Support the Students' Creativity and to Improve the Learning and Co-Learning Processes. The Polibrain Case." Pp. 4651-61 in INTED2011 Proceedings. 5th International Technology, Education and Development Conference, edited by I. Proceedings. Valencia: IATED.

Biggs, J. 2004. Calidad Del Aprendizaje Universitario. Narcea.

Glen, Roy, Christy Suciu, C. Christopher Baughn, and Robert Anson. 2015. "Teaching Design Thinking in Business Schools." International Journal of Management Education 13(2):182-92.

ICE. 2015. Proyecto de Competencias TransversalesUPV. Rúbricas.

De Miguel Molina, María, Virginia Santamarina Campos, Blanca De Miguel Molina, and María Ángeles Carabal Montagud. 2017. "Notas Móviles y Resolución de Problemas En Equipo: Del Texto Al Dibujo." in Libro de Actas IN-RED 2017 - III Congreso Nacional de Innovación Educativa y de Docencia en Red. Valencia: Universitat Politècnica València.

Muñoz González, Juan Manuel, Rocío Serrano Rodríguez, and Verónica Marín Díaz. 2014. "El Aprendizaje Colaborativo y Su Desarrollo a Través de Mapas Mentales . Una Formación Inicial Docente.” Educatio Siglo XXI 32(1):193-212.

Santamarina Campos, Virginia, María Ángeles Carabal Montagud, María De Miguel Molina, and Blanca De Miguel Molina. 2016. "La Resoluciones de Problemas a Través de Mapas Conceptuales Móviles Cooperativos." Pp. 442-56 in Libro de Actas IN-RED 2016 - II Congreso Nacional de Innovación Educativa y de Docencia en Red. Valencia: Universitat Politècnica València.

Santamarina, Virginia, María Ángeles Carabal, María de Miguel, and Blanca de Miguel. 2018. "Collaborative Visual Language for the Development of Innovative Ideas." EDULEARN18 Proceedings 1(July):535-43.

Wu-Pong, Susanna, Jogarao Gobburu, Stephen O'Barr, Kumar Shah, Jason Huber, Daniel Weiner, and American Association of Colleges of Pharmacy Graduate. 2013. "The Future of the Pharmaceutical Sciences and Graduate Education: Recommendations from the AACP Graduate Education Special Interest Group." American Journal of Pharmaceutical Education 77(4):S2. 